\title{
Time-domain study of the synchrotron radiation emitted from electron beams in plasma focusing channels
}

\author{
A. Curcio* and G. Gatti $\odot$ \\ Centro de Laseres Pulsados (CLPU), Edificio M5. Parque Científico. C/ Adaja, 8. 37185 Villamayor, Salamanca, Spain
}

(Received 8 September 2021; accepted 12 January 2022; published 10 February 2022)

\begin{abstract}
This paper sheds light on the time structure of betatron radiation, emitted by electrons that undergo betatron oscillations as they accelerate under the action of plasma wakefields. It is a common practice to assume that the betatron pulses are as short as the electron bunch length, however we show that this is not a general rule. Indeed, the betatron pulse length is affected by the betatron motion, which stretches and modulates the radiation pulses already at the source level. Propagation in a vacuum, therefore, can greatly lengthen the betatron pulses by orders of magnitude. In the wake of the above, the coherent emission of betatron radiation is studied. Coherent betatron radiation has been found to propagate in an underdense region created by ponderomotive forces, thus not suppressed by the overdense plasma absorption. This could be observed experimentally, revealing information on the acceleration process and on key beam parameters.
\end{abstract}

DOI: 10.1103/PhysRevE.105.025201

\section{INTRODUCTION}

Betatron radiation is the electromagnetic radiation emitted from electrons that undergo transverse oscillations as they accelerate under the action of three-dimensional plasma wakefields [1,2]. Synchrotronlike betatron radiation is commonly defined as temporally incoherent due to the fact that the emission spectrum can range from soft to hard x-rays (up to the $\gamma$-rays) while the shortest electron bunch length can normally range from several tens of microns down to 1 micron (or even a fraction of it). These values were experimentally determined in several experiments [3-8]. On the contrary, the spatial coherence of the betatron radiation sources has been widely demonstrated [9-13]. Although it is well known, at least theoretically, that the betatron pulses can be as short as a few femtoseconds, this duration has never been measured directly. Determining the length of these pulses could benefit experiments in which betatron radiation is used as a probe for time-resolved studies $[14,15]$. In plasma wakefield accelerators, measurement of the pulse length of betatron radiation could allow for a noninvasive measurement of the electron bunch length. In fact, the betatron radiation is emitted forward and propagates at the speed of light $c$, as approximately also relativistic electrons do. This means that under normal operating conditions, there is no significant phase-advance between the particles and the radiation on the acceleration length scale. In the same scheme of ideas, the duration of the radiation pulse is approximately equal to the electron bunch length. For the self-injection experiments, a simple formula was pro-

*acurcio@clpu.es

Published by the American Physical Society under the terms of the Creative Commons Attribution 4.0 International license. Further distribution of this work must maintain attribution to the author(s) and the published article's title, journal citation, and DOI. posed which, assuming a continuous and uniform injection of electrons in the plasma wave, gives the electron bunch length as [16]

$$
\tau_{b}=L_{\mathrm{acc}} \frac{c-v_{g}}{c^{2}}=\frac{3 L_{\mathrm{acc}} n_{e}}{2 c n_{c}},
$$

where $L_{\text {acc }}$ is the acceleration length, $v_{g}=c\left(1-3 n_{e} / 2 n_{c}\right)$ [17] is the laser group velocity in the highly nonlinear regime of laser-plasma interaction, $n_{e}$ is the electron plasma density in the region of the wakefields, and $n_{c}$ is the critical density. Equation (1) must be understood as the full width at half-maximum (FWHM), and it could overestimate the bunch length by at least a factor of $\sim 4$ [4] if the injected electron beam is high-quality and confined in the accelerating and focusing region of the plasma wave bucket (for example, obtained with a different injection scheme [18-20]). For typical parameters such as $n_{e} / n_{c} \sim 10^{-3}-10^{-2}$ and $L_{\mathrm{acc}}=1 \mathrm{~mm}$, and assuming a betatron pulse length $\tau_{\mathrm{FWHM}}$ equal to the electron bunch length, we therefore obtain $\tau_{\mathrm{FWHM}}=\tau_{b} \sim 5$ $50 \mathrm{fs}$. For longer acceleration lengths/buckets, the resulting electron bunch length can reach several hundred femtoseconds. In summary, there is a wide range of values for the betatron pulse length, which mainly depends on the electron plasma density and injection scheme. Equation (1) cannot be entirely satisfactory to understand the origin of the betatron pulse length for several reasons. The first is that it is limited to the self-injection regime. The second is that it is based on strong assumptions, which in most cases cannot be confirmed experimentally. Furthermore, strictly speaking, Eq. (1) represents the electron bunch length, which may differ from the betatron pulse length in some conditions, as shown in this paper. Numerical efforts have previously been made to provide fine tools for calculating the electromagnetic radiation [21,22]. In particular, numerical simulations of betatron radiation pulses for different wakefield acceleration regimes and laser-plasma interaction have been shown in [23]. To date, the problem of pulse duration has never been tackled analytically. 
Here we provide an analytical and time-domain approach, with the ultimate goal to obtain an illuminating expression of the betatron pulse length, scalable for different experimental configurations. We delimit the space of the parameters for which the assumption of a length of the betatron pulse equal to the electron bunch length can be true. It turned out that the betatron pulse length is determined by the electron bunch length and by the electron beam emittance. Moreover, we show how the pulse length changes dramatically as the radiation propagates from the source to the detector. In addition, it is noted that the betatron pulses are modulated by a nonlinear spectral phase, where, on average, the core of the radiation pulse contains the highest photon energies, and the neighboring tails contain the lowest radiation frequencies. Closely related to the above, we have approached a study of coherent betatron radiation, finding that it is not always suppressed by overdense plasma absorption and it might be observed experimentally. Coherent betatron radiation would allow for simpler diagnostics of the electron bunch length than time-domain measurements of incoherent $\mathrm{x}$-ray betatron pulses. Moreover, it would broaden the range of wavelengths emitted in plasma acceleration schemes. The latter would be part of the recent interest in designing compact light sources for different applications, in particular providing intense and ultrashort pulses (on the femtosecond scale) from near- to farinfrared, depending on the electron bunch length. The paper is organized as follows: in Sec. II we introduce a general formula for the evaluation of the length of the electromagnetic pulses starting from the knowledge of the spectral amplitude and the spectral phase; in Sec. III we derive an expression for the spectral amplitude and phase of the betatron radiation; in Sec. IV we apply the pulse length formula presented in Sec. II in the case of incoherent betatron radiation; in Sec. V we consider the effect of the pulse stretching induced by the propagation in a vacuum; in Sec. VI we determine the nonlinear spectralphase modulations along the betatron pulses; in Sec. VII we consider the generation and propagation of coherent betatron radiation in underdense plasmas and in a vacuum; in Sec. VIII we calculate the pulse profile and length of the coherent betatron radiation; in Sec. IX we discuss possible issues in the detection of coherent betatron radiation, such as the emission of coherent transition radiation at the plasma-vacuum boundary and the electromagnetic shadowing, i.e., the destructive interference between the radiation and the space-charge field [24]. The conclusions are reported in Sec. X.

\section{LENGTH OF A GENERIC ELECTROMAGNETIC PULSE}

The starting point will be the following equation, which relates the rms pulse length associated with any electromagnetic pulse to its spectral amplitude $|S(\omega)|$ and spectral phase $\phi(\omega)$ [25]:

$$
\tau_{\mathrm{rms}}^{2}=\int d \omega\left(\frac{d|S(\omega)|}{d \omega}\right)^{2}+\int d \omega|S(\omega)|^{2}\left(\frac{d \phi}{d \omega}\right)^{2} .
$$

The physical explanation of Eq. (2) is that the length of any electromagnetic pulse is found by a Pythagorean-like theorem, as if the square of the hypotenuse $\tau_{\mathrm{rms}}^{2}$ were the sum of the squared lengths of the two sides, the first related to the variations of the spectral amplitude and the second to the vari-

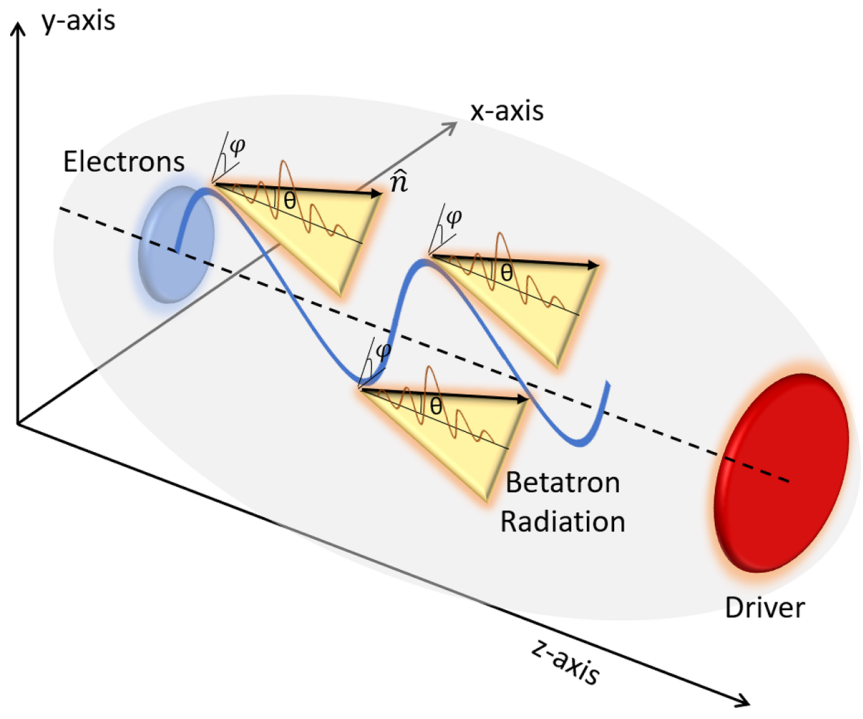

FIG. 1. Geometry of the betatron radiation emission. The shaded area indicates the plasma channel where electron oscillations occur during plasma wakefield acceleration.

ations of the spectral phase. It is worth noting that the spectral phase considered in Eq. (2) is the net phase after subtracting the contribution relative to the propagation of the center of mass of the pulse from the source to the observation point. As for the incoherent betatron radiation, the spectral amplitude is experimentally measurable, while the measurement of the spectral phase would require very sophisticated methods of phase retrieval applied to ultrashort x-ray pulses.

\section{SPECTRAL AMPLITUDE AND SPECTRAL PHASE OF BETATRON RADIATION}

From the solution of Maxwell's equations (see Appendix A), the magnetic field of frequency $\omega$ radiated at the point $\vec{r}$ by the electron current density $\vec{j}_{e}$ is

$$
\vec{H}(\vec{r}, \omega)=\vec{\nabla}_{\vec{r}} \times \int \vec{j}_{e}\left(\vec{r}^{\prime}, \omega\right) \frac{e^{i \frac{\omega}{c}\left|\vec{r}-\vec{r}^{\prime}\right|}}{4 \pi\left|\vec{r}-\vec{r}^{\prime}\right|} d^{3} r^{\prime}
$$

Since we are interested in the radiation emitted far from the source, we consider the far-field limit:

$$
\vec{H}(\hat{n}, z, \omega)=\frac{\omega}{4 \pi c} \frac{e^{i \frac{\omega z}{c}}}{z} \hat{n} \times \widetilde{\vec{j}}_{e}(\vec{k}, \omega)
$$

where $\hat{n}$ is the unit vector associated with the observation direction. The radiation wave vector is defined as $\vec{k}=\hat{n} \omega / c=$ $(\omega / c)(\sin \theta \cos \varphi \hat{x}+\sin \theta \sin \varphi \hat{y}+\cos \theta \hat{z})$. The polar and azimuth angles $\theta$ and $\varphi$ are represented in Fig. 1, and $z$ is the distance between the source and the observation point, assuming that the observation takes place around the $z$-axis.

First, we recall the spatial Fourier transform of the current density $\vec{j}(\vec{r}, t)=q \vec{v} \delta\left(\vec{r}-\vec{r}_{e}(t)\right)$ associated with a single electron of charge $q$, of velocity vector $\vec{v}$, whose motion is described by $\vec{r}_{e}(t)$ :

$$
\vec{j}(\vec{k}, t)=q \vec{v} e^{-i \vec{k} \cdot \vec{r}_{e}},
$$

where $\quad \vec{r}_{e}=\left[r_{\beta} \cos \left(\omega_{\beta} t+\psi\right)\right] \hat{x}+\left[v t+\left(v \omega_{\beta} r_{\beta}^{2} / 8 c^{2}\right)\right.$ $\left.\sin \left(2 \omega_{\beta} t+2 \psi\right)\right] \hat{z}$ [1]. In Ref. [1], the initial condition is 
that the betatron oscillation amplitude is null at the origin of time, while in the present paper it is considered different from zero (in fact, the electrons injected off-axis, i.e., those that mostly contribute to the radiation yield, oscillate with an amplitude different from zero at $t=0$ ). Finally, introducing $\psi$ takes into account an injected particle with an arbitrary phase in the plasma channel. Therefore, our betatron trajectory expression is obtained from Ref. [1] with a time shift $t \rightarrow t+\pi / 2 \omega_{\beta}$, choosing a nonzero phase lag as the initial condition for the electron dynamics and considering small betatron divergence. The betatron frequency $\omega_{\beta}$ is related to the plasma frequency $\omega_{p}$ through the Lorentz factor $\gamma$, i.e., $\omega_{\beta}=\omega_{p} / \sqrt{2 \gamma}$. The betatron amplitude is $r_{\beta}$. We have assumed motion in the $x-z$ plane, however the calculations are equally valid for the $y-z$ plane or intermediate planes, due to the symmetry of the wakefield structure. For the more general and three-dimensional case, when the motion may not even be planar with some particles following helicoidal trajectories, there are two betatron amplitudes, a horizontal $x_{\beta}$ and a vertical $y_{\beta}$ : this case is analyzed in Appendix B, but the main concepts shown below for the simpler planar case remain the same. Therefore, we argue that the formalism adopted here is sufficiently general to find the spectral phase and amplitude of the betatron radiation and to show the temporal characteristics of the betatron radiation. From now on we interpret the betatron phase $\psi$ as a dynamic variable. This approach will turn out to be useful for evaluating the radiation from a bunch of particles. This means that for a $\psi$ fixed along the betatron trajectory, we can select a short interval of time where, locally, the argument of the imaginary exponential function in Eq. (5) can be expanded as

$$
\vec{j}(\vec{k}, t) \simeq q \vec{v} e^{i \theta_{\beta}^{2} \frac{\omega \omega_{\beta}^{2} t^{3}}{6} f^{2}(\psi)-i \omega\left(1-\frac{1}{2 \gamma^{2}}-\frac{\theta^{2}}{2}+\frac{\theta_{\beta}^{2} f^{2}(\psi)}{4}\right) t} e^{i g(\psi)} .
$$

With a standard procedure in polynomial calculus [26], the quadratic phase term in time has been ruled out with a suitable time shift of the order of $1 / \omega_{\beta}$, in order to facilitate the derivation. This is always allowed when the number of betatron oscillations is at least half. Please note that we have used $\theta \lesssim \theta_{\beta}=\omega_{\beta} r_{\beta} / c \ll 1$, with $\theta_{\beta}$ being the divergence of the betatron source, and furthermore we already have considered $\psi \ll 1$, justified by Eq. (13) later in the calculations in this section. We have also defined

$$
f^{2}(\psi)=\cos (2 \psi)
$$

and

$$
g(\psi)=\frac{\omega}{2 \omega_{\beta}} \tan (2 \psi) .
$$

Equation (6) must be interpreted as the current associated with a particle emitting in $\psi$ phase of the betatron trajectory. The Fourier transform of Eq. (6) gives the quantity we are interested in, for the calculation of betatron radiation by means of Eq. (4):

$$
\begin{aligned}
\widetilde{\vec{j}}_{x}(\vec{k}, \omega)= & 2 \pi i q r_{\beta}\left(\frac{2 \omega_{\beta}}{\omega \theta_{\beta}^{2} f^{2}(\psi)}\right)^{\frac{2}{3}} \mathrm{Ai}^{\prime}\left[\left(\frac{3 \omega}{4 \omega_{c}(\psi)}\right)^{\frac{2}{3}}\left(1+\gamma^{2} \theta^{2}-\frac{\gamma^{2} \theta_{\beta}^{2} f^{2}(\psi)}{2}\right)\right] e^{i g(\psi)} \\
& -2 \pi q r_{\beta} \sin (\psi)\left(\frac{2 \omega_{\beta}}{\omega \theta_{\beta}^{2} f^{2}(\psi)}\right)^{\frac{1}{3}} \mathrm{Ai}\left[\left(\frac{3 \omega}{4 \omega_{c}(\psi)}\right)^{\frac{2}{3}}\left(1+\gamma^{2} \theta^{2}-\frac{\gamma^{2} \theta_{\beta}^{2} f^{2}(\psi)}{2}\right)\right] e^{i g(\psi)}, \\
\widetilde{\vec{j}}_{y}(\vec{k}, \omega)= & 0, \\
\widetilde{\vec{j}}_{z}(\vec{k}, \omega)= & 2 \pi q v\left(\frac{2}{\omega \omega_{\beta}^{2} \theta_{\beta}^{2} f^{2}(\psi)}\right)^{\frac{1}{3}} \mathrm{Ai}\left[\left(\frac{3 \omega}{4 \omega_{c}(\psi)}\right)^{\frac{2}{3}}\left(1+\gamma^{2} \theta^{2}-\frac{\gamma^{2} \theta_{\beta}^{2} f^{2}(\psi)}{2}\right)\right] e^{i g(\psi),}
\end{aligned}
$$

where we introduced the Airy function of the first kind $\operatorname{Ai}(x)$ and its derivative $\operatorname{Ai}^{\prime}(x)$. The $\widetilde{\vec{j}}_{x}$ component gives rise to synchrotronlike radiation polarized in the plane of the betatron oscillation, while the $\widetilde{\vec{j}}_{z}$ component gives rise to a polarization perpendicular to that plane. Finally, the spectral angular distribution of the radiated energy $E$ emitted inside the solid angle $\Omega$ as incoherent betatron radiation will be

$$
\frac{d^{2} E}{d \omega d \Omega}=\frac{\mu_{0} \omega^{2}}{16 \pi^{3} c}\left(\left.\widetilde{\vec{j}}_{x}(\vec{k}, \omega)\right|^{2}+\theta^{2}\left|\widetilde{\vec{j}}_{z}(\vec{k}, \omega)\right|^{2}\right),
$$

where $\mu_{0}=1 / \varepsilon_{0} c^{2}$ is the magnetic permeability of vacuum. The definition of critical frequency $\omega_{c}(\psi)$ is

$$
\omega_{c}(\psi)=\frac{3}{2} \gamma^{3} \omega_{\beta} \theta_{\beta} f(\psi) .
$$

The above equation means that the critical frequency dynamically changes along the betatron trajectory. The integral over the solid angle of Eq. (10) can be performed:

$$
\left|S_{\beta}(\omega)\right|^{2}(\psi)=\frac{d E}{d \omega} \simeq \frac{\sqrt{3}}{4 \pi} \frac{q^{2} \gamma}{\varepsilon_{0} c} \frac{\omega}{\omega_{c}(\psi)} \int_{\frac{\omega}{\omega_{c}(\psi)}}^{\infty} K_{5 / 3}(x) d x,
$$

where $\left|S_{\beta}(\omega)\right|(\psi)$ is the spectral amplitude of the betatron radiation for an electron associated with the betatron phase $\psi$. The integral over the $\omega$ frequency of Eq. (12) instead gives

$$
E(\psi)=\frac{q^{2} \gamma^{4}}{3 \varepsilon_{0} c} \theta_{\beta} \omega_{\beta} f(\psi) .
$$

Equation (13) states that the emitted energy oscillates along the betatron trajectory. As shown in Fig. 2, most of the energy is radiated for the betatron phases $\psi=n \pi$ with $n=$ $0,1,2, \ldots$, in agreement with the saddlepoint approximation described in Ref. [27]. Therefore, for an approximate evaluation of the spectral phase, we consider $\psi \simeq 0$. For 


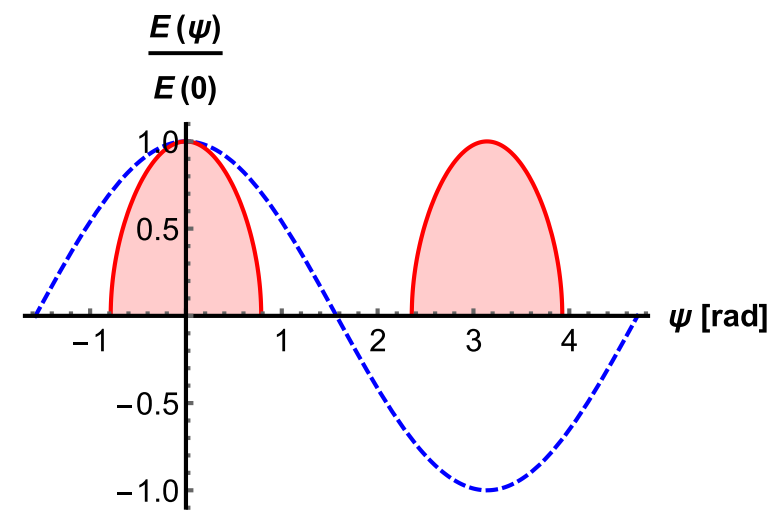

FIG. 2. The dashed blue line represents the betatron amplitude (normalized units) of a particle that emits radiation in the phase $\psi$ of its trajectory. The red line encloses the region where energy is radiated by the particle, the level of the curve indicating the amount of radiation. The emission of betatron radiation occurs mainly near the turning points of the betatron oscillation.

the spectral amplitude that absorbs all the phase terms but not $g(\psi)$ in Eq. (6), the spectral phase of the betatron radiation field (including the propagation of the barycenter of the pulse), considering also Eq. (4) and an observation point $R=\sqrt{x^{2}+z^{2}}$ such that $z \gg x$, is

$$
\Phi(\omega) \simeq \frac{\omega}{c}\left(\sqrt{R^{2}-x^{2}}\right)+g(\psi \simeq 0) \simeq \frac{\omega}{c}\left(R-r_{\beta} \theta_{\beta}-c t_{0}\right),
$$

where we used $x^{2}=r_{\beta}^{2}+2 r_{\beta} \theta_{\beta} z+\theta_{\beta}^{2} z^{2}$ for the light rays exiting the source with divergence $\theta_{\beta}$ and also the fact that for an arbitrary particle delayed by $t_{0}$ with respect to the reference particle placed at $t=0$, the betatron phase lag can be expressed as $\psi=-\omega_{\beta} t_{0}$.

\section{PULSE LENGTH AND TIME STRUCTURE OF INCOHERENT BETATRON RADIATION}

We recall that in Sec. II, the spectral phase has been defined as $\phi(\omega)=\Phi(\omega)-\omega R / c$, i.e., subtracting the propagation time of the pulse as a whole. Let us now evaluate Eq. (2) using the normalization rule $\int d \omega\left|S_{\beta}(\omega)\right|^{2}=1$ and considering the maximum emission phase $\psi=0$. Under usual experimental conditions, the first term on the right side of Eq. (2) is of the order of $\sim 1 / \omega_{c}^{2} \ll \omega_{\beta}^{2} r_{\beta}^{4} / c^{4}, t_{0}^{2}$, so it is negligible compared to the second term. To obtain a reliable expression for the betatron pulse length, the betatron radius, divergence, and delay with respect to the reference particle must be considered over the entire ensemble of electrons. We therefore define the rms spectral phase $\phi_{\mathrm{rms}}(\omega)$ as the phase $\phi(\omega)$ for which the parameters $\theta_{\beta}, r_{\beta}$, and $t_{0}$ undergo a process of root mean square (rms) calculated over the beam distributions. $\Theta\left(\theta_{\beta}\right)$, $R\left(r_{\beta}\right)$, and $T\left(t_{0}\right)$ are, respectively, the distribution functions of the betatron divergence, of the betatron radius, and of the longitudinal electron position along the bunch:

$$
\phi_{\mathrm{rms}}=-\omega\left(\tau_{e}+\frac{\epsilon}{c}\right) .
$$

The rms betatron pulse length is found by inserting Eq. (15) into Eq. (2) in place of $\phi$ while neglecting the first term of the latter equation for the reasons discussed above:

$$
\tau_{\mathrm{rms}}=\tau_{e}+\frac{\epsilon}{c} .
$$

In Eq. (16) we recognized the rms electron beam emittance $\epsilon=\hat{\theta}_{\beta} \sigma$, with $\hat{\theta}_{\beta}$ and $\sigma$ the rms electron beam divergence and the rms electron beam size, respectively. Indeed, the product of beam size and beam divergence at a turning point is equal to the beam emittance since the correlation in the phase space is zero. Moreover, according to Ref. [28], $\epsilon$ should be multiplied by a correction factor 2 in Eqs. (15) and (16). This factor would derive from the expression of the effective rms betatron amplitude over one oscillation cycle. Nevertheless, since most of the photon emission is confined around the turning point of the betatron trajectory, the real correction factor is closer to 1 . Finally, the rms betatron pulse length consists of two terms, the first trivial, related to the electron bunch length, and the second related to the phase retardation at the observation point due to the finite emittance of the source. The retardation term accounts for the dephasing occurring among different source points in the plane transverse to the emission, i.e., among different electrons distributed in the plane transverse to the propagation. In other words, within the observation cone determined by $\theta_{\beta}$, the rays emitted by extremal points of the source will accumulate a relative delay inducing a measurable pulse stretching. Equation (16) must be considered when the electron bunch length is comparable to the electron beam emittance, i.e., for ultrashort beams of poor quality. For example, an electron beam emittance $\epsilon=220 \mathrm{~nm}$ would correspond to an elongation of the rms pulse length of 1 fs: for very short bunch lengths, the effect of the emittance is relevant and must be taken into account. In conclusion, and for the sake of clarity, the electron bunch length and the beam emittance can evolve during the acceleration process, therefore the final betatron radiation pulse carries integrated information of such beam dynamics. It is worth noting that in Ref. [23], a case is studied in which the FWHM duration of the betatron pulse is shorter than the FWHM bunch length. On the contrary, the analysis proposed in this paper considers rms and integrated (along the acceleration path) quantities. In this latter framework, the case of an incoherent betatron pulse shorter than the electron bunch is physically suppressed at relativistic energies.

\section{STRETCHING OF THE BETATRON PULSES PROPAGATING IN A VACUUM}

Equation (16) is valid only at the source level. The propagation in a vacuum of the betatron pulse can affect its length. This can be seen expanding the spectral phase while keeping the corrections in the order of $\hat{\theta}_{\beta}^{2}$ :

$$
\phi_{\mathrm{rms}}(\omega)=-\frac{\omega}{c}\left(c \tau_{e}+\epsilon+\frac{\hat{\theta}_{\beta}^{2} z}{2}\right),
$$

which yields a $z$-dependent expression of the rms betatron pulse length via Eq. (2):

$$
\tau_{\mathrm{rms}}(z)=\tau_{e}+\frac{\epsilon}{c}+\frac{\hat{\theta}_{\beta}^{2} z}{2 c} .
$$




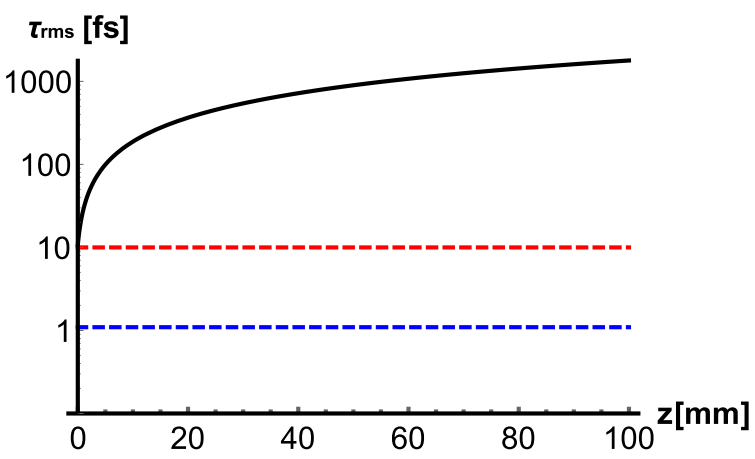

FIG. 3. Comparison between the betatron pulse $\tau_{\text {rms }}$ (black line) stretching during propagation in a vacuum, and the contribution from the bunch length $\tau_{e}$ (dashed red) and from the beam emittance $\epsilon / c$ (dashed blue) at the source level. Used parameters: $\epsilon=220 \mathrm{~nm}, \tau_{e}=$ $10 \mathrm{fs}$, and $\omega_{\beta}=7.3 \times 10^{12} \mathrm{~s}^{-1}$ corresponding to $n_{e}=10^{19} \mathrm{~cm}^{-3}$ and $\gamma=300$, i.e., to a divergence $\hat{\theta}_{\beta}=70 \mathrm{mrad}$.

For ultrashort pulses, as in the case of betatron radiation, the propagation in a vacuum can be responsible for an important stretching of the pulse length from its initial value in the plasma, connected only with the electron bunch length and to a lesser extent to the electron beam emittance. For example, with the parameters used in Fig. 3, the betatron pulse is stretched by two orders of magnitude in less than $1 \mathrm{~m}$ of propagation in a vacuum. For smaller betatron divergence and better beam emittance, the elongation is less critical and the contribution from the beam emittance is completely negligible, as shown in Fig. 4. Nevertheless it is extremely remarkable that the pulse length measured at a certain distance from the source can be significantly longer than the electron bunch length at the source (also the electron bunch can elongate as it propagates in a vacuum with a divergence). A simple physical explanation for the elongation in a vacuum is given below. Indeed, in the propagation of diverging ultrashort pulses, this effect is to be expected. A photon propagating in a vacuum over a distance $L$, on axis, would correspond to a trajectory $z=c t$, i.e., to an arrival time $t_{1}=L / c$.

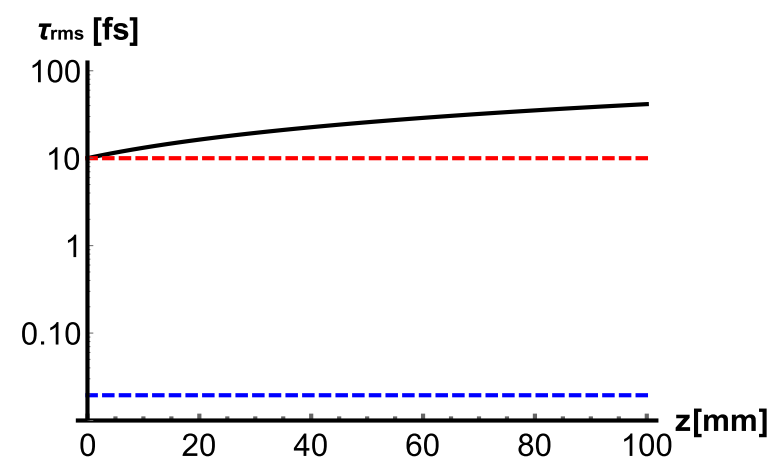

FIG. 4. Comparison between the betatron pulse $\tau_{\text {rms }}$ (black line) stretching during propagation in a vacuum, and the contribution from the bunch length $\tau_{e}$ (dashed red) and from the beam emittance $\epsilon / c$ (dashed blue) at the source level. Used parameters: $\epsilon=3.9 \mathrm{~nm}, \tau_{e}=$ $10 \mathrm{fs}$, and $\omega_{\beta}=7.3 \times 10^{12} \mathrm{~s}^{-1}$ corresponding to $n_{e}=10^{19} \mathrm{~cm}^{-3}$ and $\gamma=300$, i.e., to a divergence $\hat{\theta}_{\beta}=10 \mathrm{mrad}$.

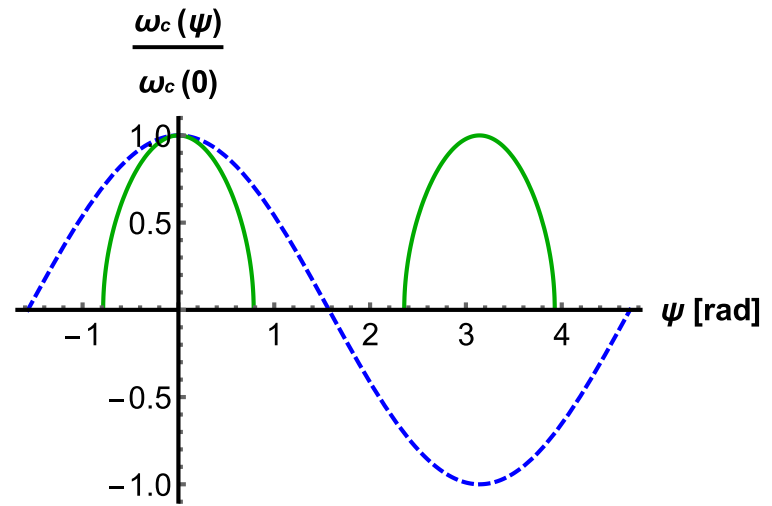

FIG. 5. Normalized critical frequency (green line) for an electron bunch emitting betatron radiation. The betatron oscillation (dashed blue, normalized units) is reported in the same plot. The frequency modulation is approximately quadratic close to the phases $\psi=0, \pi$ of maximum emission.

A photon propagating with a small angle $\theta \ll 1$, corresponding to a trajectory $z=v_{z} t=c \cos \theta t \simeq c t\left(1-\theta^{2} / 2\right)$, has an arrival time of $t_{2}=L / c\left(1-\theta^{2} / 2\right)$ at the same plane. The time difference (responsible for the pulse elongation) between the two photons is $t_{2}-t_{1} \simeq \theta^{2} L / 2 c$, consistent with Eq. (18), and the more complete one, Eq. (B4). To conclude, the short pulse lengths could be restored by using a pinhole/aperture or proper focusing optics in front of an irradiated sample/detector, but at the expense of photon flux or possibly introducing other effects like, e.g., longitudinal dispersion.

\section{NONLINEAR PHASE MODULATIONS}

The betatron oscillations transduce into a nonlinear spectral phase modulation along the betatron radiation pulse. Indeed, the dominant frequency along the radiation pulse is given by Eq. (11). Passing through the regions of maximum emission, the electron bunch emits a radiation frequency that is lower if coming from its head and its tail and higher if emitted from its center, as shown in Fig. 5. The above trend of the critical frequency along the betatron pulse is perfectly consistent with the numerical results shown in Ref. [23], where it is shown that the critical frequency is maximum at the peak of the pulse and it decays along the front and the rear of it. This information would be quite relevant when implementing diagnostic techniques of the betatron pulse length and timeresolved experiments whose reliability depends strongly upon the frequency-time correlation along the radiation pulses. All the above applies to the incoherent betatron radiation, and, after proper treatment of the transduction of the pulse length from electrons to radiation, it can simply be extended to the coherent part of the spectrum, as shown below.

\section{PROPAGATION OF COHERENT BETATRON RADIATION}

Coherent betatron radiation (CBR) would extend to $\omega=$ $1 / \tau_{e}$ and beyond, which, for $\tau_{e}=1 \mathrm{fs}$, would mean a coherence bandwidth up to $\gtrsim 0.66 \mathrm{eV}$ (or even above $1 \mathrm{eV}$ for a 
microstructured electron bunch [8]). The coherent spectrum would cover much lower frequencies than the incoherent spectrum, normally ranging from a few to many keV. Therefore, it would extend to the NIR-visible region. For the sake of rigor, Eq. (4) should be modified for the presence of the plasma medium in such a way that

$$
\vec{H}(\hat{n}, z, \omega)=\frac{\sqrt{\varepsilon(\omega)} \omega}{4 \pi c} \frac{e^{i \frac{\sqrt{\varepsilon(\omega)} \omega z}{c}}}{z} \hat{n} \times \widetilde{\vec{j}}_{e}(\vec{k}, \omega),
$$

where $\vec{k}=\sqrt{\varepsilon(\omega)} \omega \hat{n} / c$, and the plasma dielectric function is defined as

$$
\varepsilon(\omega)=1-\frac{\omega_{p}^{2}}{\omega^{2}}
$$

From direct inspection of Eq. (19) it is clear that any frequency $\omega<\omega_{p}$ propagates as an evanescent wave, hence the plasma is said to be overdense. For an electron plasma density $n_{e}=1 \times 10^{19} \mathrm{~cm}^{-3}$, the plasma frequency is $\omega_{p} \simeq 0.18 \mathrm{PHz}$, corresponding to a photon cutoff energy of $\sim 0.12 \mathrm{eV}$ (in a wavelength $10.7 \mu \mathrm{m}$ ). This means that CBR photons with energy $\mathrm{eV}$ (or a fraction thereof), propagating in the region of electron density $n_{e}$, should not be suppressed for such experimental conditions, and therefore CBR should be observable. Furthermore, the situation is even more favorable due to the fact that within the emission region, the electron plasma density is strongly suppressed by the ponderomotive forces. This means that the dielectric function of the plasma must be evaluated replacing the electron plasma frequency $\omega_{p}$ with the ion plasma frequency $\omega_{p, i}$, which is over one order of magnitude smaller. As for the propagation out of the plasma, technically speaking the temporal coherence of the betatron radiation pulse, inversely proportional to the spectral bandwidth, is not affected by the stretching in the vacuum, since the latter does not introduce longitudinal dispersion. The divergence is such that it angularly disperses the pulse, which locally maintains the same degree of temporal coherence. Eventually, refocusing the radiation beam could restore pulse length and coherence, but it may introduce other effects like interference or longitudinal dispersion. What is affected by the divergence is also the degree of spatial coherence, which is inversely proportional to the source divergence, although it also increases with the radiated wavelength, so the coherent betatron source remains nevertheless highly spatially coherent. Indeed, the divergence of $\mathrm{CBR}$ is not $\theta_{\beta}$ but it is frequency-dependent [1]:

$$
\theta_{d}=\frac{1}{\gamma}\left(\frac{\omega_{c}(0)}{\omega}\right)^{1 / 3}
$$

Given typical values $\theta_{\beta}=10 \mathrm{mrad}, \gamma=300$, and $\omega_{\beta}=7.3 \times$ $10^{12} \mathrm{~s}^{-1}$, as in Fig. 4, the above formula gives $\theta_{d}=39 \mathrm{mrad}$ at the radiated wavelength $1 \mu \mathrm{m}, \theta_{d}=83 \mathrm{mrad}$ for $10 \mu \mathrm{m}$ radiation, and $\theta_{d}=130 \mathrm{mrad}$ for $100 \mu \mathrm{m}$ radiation. It is evident that coherent betatron radiation is more divergent than incoherent betatron radiation, and that it could suffer from more severe pulse elongation.

\section{TEMPORAL PROFILE OF COHERENT BETATRON PULSES}

Referring to the method adopted in Sec. IV, where the incoherent current was $\vec{j}_{e} \propto \sqrt{N_{e}} \exp \left(-i \omega \tau_{e}\right)$, for the coherent wavelengths the current density becomes

$$
\vec{j}_{e}(\vec{k}, \omega)=q v N_{e} P(\vec{k}, \omega) \hat{z}=q v \rho(\vec{k}, \omega) \hat{z},
$$

where we introduced the so-called form factor $\rho(\vec{k}, \omega)=$ $N_{e} P(\vec{k}, \omega)$, with $N_{e}$ the number of electrons in the emitting bunch, and $P(\vec{k}, t)$ the temporal profile of the electron current density. The $\tau_{e}$-dependent spectral phase term of the betatron radiation field disappears, now embedded in the spectral amplitude. Thus, for coherent betatron radiation we obtain

$$
\phi_{\mathrm{rms}}=-\frac{\omega \epsilon}{c}
$$

and

$$
\frac{d|S|}{d \omega}=\frac{\left|S_{1}\right|}{\omega_{c}}+\left|S_{2}\right| \tau_{e} \simeq\left|S_{2}\right| \tau_{e},
$$

where we again considered $\omega_{c}$ much larger than all the other frequencies of the problem, justified by normal working conditions $\left(\omega_{c} \tau_{e} \gg 1\right)$. We have defined $\left|S_{1}\right| / \omega_{c}$ and $\left|S_{2}\right| \tau_{e}$ as the slowly varying and rapidly varying parts of the derivative of the spectral amplitude of betatron radiation $|S|$, which now contains information on both coherent and incoherent photons. The coherent betatron pulse length is finally found via Eq. (2):

$$
\tau_{\mathrm{rms}}=\alpha \tau_{e}+\frac{\epsilon}{c}
$$

where $\alpha$ is a factor ranging between 0 and 1, which depends on the temporal profile of the electron bunch. The definition of this parameter is $\alpha=\left[\int d \omega\left|S_{2}(\omega)\right|^{2} / \int d \omega|S(\omega)|^{2}\right]^{1 / 2}$, where the normalization rule $\int d \omega|S(\omega)|^{2}=1$ holds. Recall that Eq. (25) refers to the betatron pulse length at the source, while the $z$-dependent term appearing in Eq. (18) must be added when evaluating the pulse length far from the source, at a distance $z$ from it. When passing from incoherent to coherent radiation, the contributions from the beam emittance and from the propagation in a vacuum do not change. The only different term is the one related to the electron bunch length, carrying information on the bunch form factor. The temporal profile $T_{\beta}(t)$ of the coherent betatron pulses in the conditions where the emittance is negligible in Eq. (25), as well as close enough to the source for avoiding pulse stretching, is given by

$$
T_{\beta}(t) \propto\left|\frac{1}{2 \pi} \int d \omega S(\omega) e^{-i \omega t}\right|^{2},
$$

where the symbol $\propto$ has been used to denote the fact that the left and right sides of Eq. (19) are equal up to a normalization constant. Since $|S(\omega)| \simeq\left|S_{\beta}(\omega)\right| P(\omega)$, the incoherent spectral amplitude $\left|S_{\beta}\right|$ acts as a transfer function between the electron bunch (characterized by $P$ ) and the radiation pulse (characterized by $|S|$ ). It is possible to see that this transfer function can modify the shape of the pulse when passing from the particles to the radiation, and that for coherent radiation the pulse length is $<\tau_{e}$, i.e., $\alpha<1$, due to the fact that $T_{\beta}(t) \propto j^{2}(t)$. The above is shown in Fig. 6, obtained through Eq. (26), where the radiation profile is similar to the square 


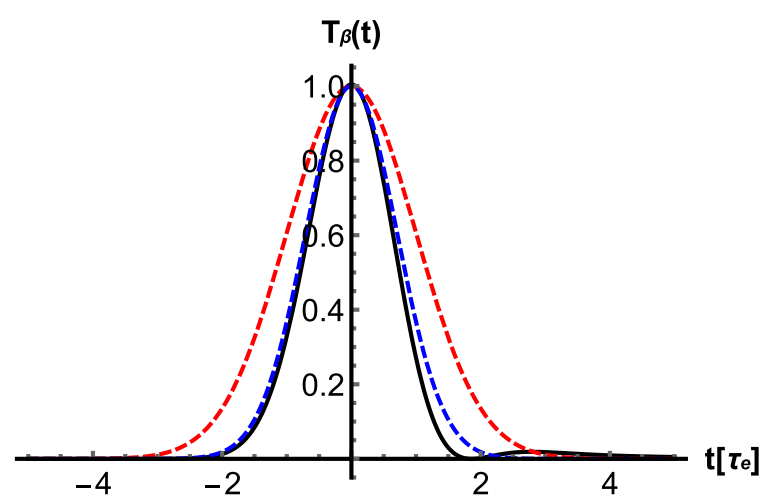

FIG. 6. Temporal profile of a CBR pulse (black line) corresponding to a Gaussian current density profile with rms length $\tau_{e}$. The timescale is expressed in units of $\tau_{e}$. For comparison, the normalized current density profile is reported (dashed red line) as well as the square of it (dashed blue line).

of the current density profile except for a small tail at late times. In Appendix C, it is shown that for a Gaussian current, $\alpha=\sqrt{2 / 3}$. In general, the electron bunch may be composed of a microstructured pattern with more than one dominant frequency in the spectrum: in that case, $T_{\beta}$ would inherit the same structure. The temporal profile is determined by the coherent part of the spectrum, which extends beyond $\omega=1 / \tau_{e}$. The coherent spectrum can also show resonances at frequencies $\omega \gg 1 / \tau_{e}$ in the presence of microstructures in the electron bunch profile. Such modulations could also be artificially induced via electromagnetic pulses [29]. It is interesting to estimate the number of photons emitted in the coherent part of the spectrum compared to the number of photons emitted in the incoherent region. This can be achieved by recalling that the low-frequency scaling for synchrotronlike emission is $\left|S_{\beta}(\omega)\right|^{2} \sim\left(\omega / \omega_{c}\right)^{1 / 3}$ [see Eq. (10) and Refs. [30,31]], while we can consider $\left|S_{\beta}(\omega)\right|^{2} \sim 1$ for frequencies $\omega \sim \omega_{c}$. Therefore, the number of coherent photons will be $N_{\text {coh }} \propto$ $N_{e}^{2}\left(\omega / \omega_{c}\right)^{1 / 3}$ while the number of incoherent photons will be $N_{\text {inc }} \propto N_{e}$. The ratio between the two photon populations is $N_{\text {coh }} / N_{\text {inc }} \sim N_{e}\left(\omega / \omega_{c}\right)^{1 / 3}$. For $\omega$ corresponding to $1 \mathrm{eV}$ and $\omega_{c}$ corresponding to $5 \mathrm{keV}$, we obtain $\left(\omega / \omega_{c}\right)^{1 / 3} \sim 6 \%$. For $N_{e}=10^{8}$, the coherent emission is at least six orders of magnitude more intense than the incoherent counterpart.

\section{THE TRANSITION RADIATION ISSUE}

One problem that can occur in the CBR detection is that CBR can get confused with coherent transition radiation (CTR) at the plasma-vacuum boundary. The criterion for determining whether CBR is more important than CTR is based on comparing radiation yields. The radiation yield of CBR can be estimated via Eq. (13) after multiplying by the number of turning points along the acceleration path, which is roughly $2 L_{\mathrm{acc}} \omega_{\beta} / c$ :

$$
Y_{\mathrm{CBR}}=\frac{2 L_{\mathrm{acc}} q^{2} \gamma^{4} \theta_{\beta} \omega_{\beta}^{2}}{3 \varepsilon_{0} c^{2}} .
$$

The yield of CTR for a very sharp plasma-vacuum transition is obtained as [32]

$$
Y_{\mathrm{CTR}}=\frac{q^{2} \gamma \omega_{p}}{12 \pi \varepsilon_{0} c} .
$$

The ratio between the two yields is

$$
Y=\frac{Y_{\mathrm{CBR}}}{Y_{\mathrm{CTR}}}=\frac{24 \pi L_{\mathrm{acc}} \gamma^{3} \theta_{\beta} \omega_{\beta}^{2}}{3 \omega_{p} c}=\frac{12 \pi L_{\mathrm{acc}} \gamma^{2} \theta_{\beta} \omega_{p}}{3 c} .
$$

For typical experimental parameters of electron plasma acceleration, evidently $Y \gg 1$. Moreover, for sufficiently smooth plasma down-ramps (decaying over a distance comparable to or greater than the CTR wavelengths), the CTR is further suppressed compared to the CBR. Due to the finite transverse size of the CTR source, a correction due to coherent diffraction radiation (CDR), very critical at low frequencies [33,34], should also be considered. The above confirms once again the feasibility of a CBR detection experiment, which from a practical point of view could ease the determination of the betatron pulse length, as well as of the electron bunch length. To get information on coherent radiation yields, we have used the expression of incoherent radiation yields [Eqs. (27) and (28) refer to a single emitter]. This is justified by the fact that the incoherent yield is proportional to the intrinsic probability of the emission process occurring, both incoherently and coherently. A more rigorous treatment would require knowledge of the form factor, such that the coherent yields can be compared directly. Nevertheless, the result for the relative yield $Y$ would be very similar, given the fact that the coherent region of the spectrum would be approximately the same for the two mechanisms (CBR and CTR), but not the probability of photon emission. Finally, the spectral detection of CBR would directly reveal microbunching and/or multibunch structures. Bending the electrons before the radiation detector could also solve electromagnetic shadowing problems, separating the electrons from the radiation [24] and allowing the measurement of the latter while avoiding destructive interference.

\section{CONCLUSIONS}

The temporal characteristics of the betatron radiation emitted by relativistic electrons accelerated in the plasma channels have been reviewed with a fully analytic approach. The study revealed interesting features, such as the fact that the finite duration of the betatron radiation pulses is determined, in the general case, by the electron bunch length, as already known from previous literature, but also by the beam emittance. We demonstrated the presence of a nontrivial frequency-time correlation along the pulse, also visible in the simulation results reported in Ref. [23]. We discussed the issue of the pulse elongation during the propagation in a vacuum, which, through the divergence of the source, acts by delaying the external rays relative to the internal ones by a dramatic amount. Such stretching of the betatron pulses can reach several orders of magnitude on typical experimental distances. This phenomenology is completely described by Eq. (18) for the 1D case, while the 3D case is described in Appendix B resulting in Eq. (B4). This last equation can explain the value measured in Ref. [16], although the experiment reported therein was limited by the diagnostic apparatus. 
Furthermore, we highlighted the relevance and the characteristics of temporally coherent betatron radiation. In fact, $\mathrm{CBR}$ in the visible/infrared/THz domain (according to the interaction regimes) can be emitted and propagated outside the source, making possible a new class of beam diagnostics and/or compact light sources. In conclusion, the paper sheds light on the temporal properties of betatron radiation pulses, aiming to obtain information and scalable formulas for diverse experimental conditions. The work is meant to be of interest to researchers in the field of electron beam diagnostics for plasma accelerators as well as for the radiation users who leverage betatron pulses to perform time-domain studies of dynamic samples.

\section{ACKNOWLEDGMENTS}

The research leading to these results has received funding from LASERLAB-EUROPE V (Grant Agreement No. 871124, European Union Horizon 2020 research and innovation program).

\section{APPENDIX A: DERIVATION OF EQ. (3)}

The Maxwell equation for the magnetic field $\vec{H}$ is

$$
\vec{\nabla} \times \vec{H}=\vec{j}+\varepsilon_{0} \partial_{t} \vec{E},
$$

where $\vec{j}$ is an electric current density and $\vec{E}$ is the electric field. We first consider the curl of Eq. (A1):

$$
\nabla^{2} \vec{H}=-\vec{\nabla} \times \vec{j}-\varepsilon_{0} \partial_{t} \vec{\nabla} \times \vec{E} .
$$

Furthermore, using the Maxwell equation for $\vec{E}$ :

$$
\vec{\nabla} \times \vec{E}=-\mu_{0} \partial_{t} \vec{H}
$$

and then switching to the frequency domain, we obtain

$$
-\frac{\omega^{2}}{c^{2}} \vec{H}-\nabla^{2} \vec{H}=\vec{\nabla} \times \vec{j} .
$$

The retarded Green function of Eq. (A4) is [31]

$$
\Phi\left(\vec{r}-\vec{r}^{\prime}, \omega\right)=\frac{e^{i \frac{\omega}{c}\left|\vec{r}-\vec{r}^{\prime}\right|}}{4 \pi\left|\vec{r}-\vec{r}^{\prime}\right|} .
$$

Thus, considering the curl of the current density as a source term, the final solution of Eq. (A4), i.e., the magnetic field $\vec{H}$ radiated by the current density $\vec{j}$, is

$$
\vec{H}(\vec{r}, \omega)=\vec{\nabla}_{\vec{r}} \times \int_{V_{R}} \Phi\left(\vec{r}-\vec{r}^{\prime}, \omega\right) \vec{j}\left(\vec{r}^{\prime}, \omega\right) d^{3} r^{\prime} .
$$

\section{APPENDIX B: SPECTRAL PHASE AND AMPLITUDE IN THE THREE-DIMENSIONAL CASE}

The 3D parametrization of betatron trajectory is

$$
\begin{aligned}
x(t)= & x_{\beta} \cos \left(\omega_{\beta} t+\psi_{x}\right), \\
y(t)= & y_{\beta} \cos \left(\omega_{\beta} t+\psi_{y}\right), \\
z(t)= & v t-\frac{v}{8 c}\left[x_{\beta} \theta_{x} \sin \left(2 \psi_{x}\right)+y_{\beta} \theta_{y} \sin \left(2 \psi_{y}\right)\right] \\
& +\frac{v}{8 c}\left[x_{\beta} \theta_{x} \sin \left(2 \psi_{x}+2 \omega_{\beta} t\right)\right. \\
& \left.+y_{\beta} \theta_{y} \sin \left(2 \psi_{y}+2 \omega_{\beta} t\right)\right]
\end{aligned}
$$

where $\theta_{x, y}$ and $\psi_{x, y}$ are the betatron divergence and the phaselag in the horizontal and vertical plane, respectively. Similarly to Sec. III, we obtain

$$
\begin{aligned}
\vec{j}(\vec{k}, t) \simeq & q \vec{v} e^{i \frac{\omega r_{\beta}^{2} \omega_{\beta}^{4} t^{3}}{6 c^{2}}\left[\cos ^{2} \varphi \cos \left(2 \psi_{x}\right)+\sin ^{2} \varphi^{2} \cos \left(2 \psi_{y}\right)\right]} \\
& \times e^{-i \omega t\left(1-\frac{1}{2 \gamma^{2}}-\frac{\theta^{2}}{2}+\frac{\gamma^{2} \theta_{\beta}^{2}}{2}\left[\cos ^{2} \varphi \cos \left(2 \psi_{x}\right)+\sin ^{2} \varphi^{2} \cos \left(2 \psi_{y}\right)\right]\right)} \\
& \times e^{\frac{i \omega}{2 \omega}\left[\tan \left(2 \psi_{x}\right)+\tan \left(2 \psi_{y}\right)\right]}
\end{aligned}
$$

The nontrivial dependence upon $\varphi$ now makes the integral over the solid angle less straightforward, thus we define

$$
\left|S_{\beta}(\omega)\right|^{2}=\int_{0}^{2 \pi} \int_{0}^{\pi} d \varphi d \theta \sin \theta \frac{d^{2} E}{d \omega d \Omega},
$$

where $d^{2} E / d \omega d \Omega$ follows from Eqs. (10) and (B2). The resulting rms pulse length of betatron radiation is finally

$$
\tau_{\mathrm{rms}}(z)=\alpha \tau_{e}+\frac{\sqrt{\epsilon_{x}^{2}+\epsilon_{y}^{2}}}{c}+\frac{\hat{\theta}_{\beta}^{2} z}{2 c}
$$

where $\epsilon_{x}$ and $\epsilon_{y}$ are the horizontal and vertical rms emittances, respectively, and we recall that $z$ is the distance from the betatron source to the detector. $\alpha=1$ for incoherent radiation and $0<\alpha<1$ for coherent radiation.

\section{APPENDIX C: DURATION OF COHERENT BETATRON PULSES GENERATED BY GAUSSIAN ELECTRON BUNCHES}

Given a Gaussian electron current density, the Fourier transform of the bunch profile associated with it is

$$
P(\omega)=\frac{\tau_{e}}{\sqrt{2 \pi}} e^{-\frac{\omega^{2} \tau_{e}^{2}}{2}} .
$$

The betatron spectral amplitude is by consequence

$$
|S(\omega)|=\frac{1}{\sqrt{N_{S}}}\left(\frac{\omega}{\omega_{c}}\right)^{1 / 6} \frac{\tau_{e}}{\sqrt{2 \pi}} e^{-\frac{\omega^{2} \tau_{e}^{2}}{2}},
$$

where $N_{S}$ is a normalization constant such that $\int d \omega|S(\omega)|^{2}=1$. The rapidly varying part of the spectral amplitude is found by taking the derivative of Eq. (C2) while neglecting the term $\propto 1 / \omega_{c}$,

$$
\left|S_{2}(\omega)\right|=-\omega \tau_{e}|S(\omega)| .
$$

Via its definition, the $\alpha$ parameter is calculated as

$$
\alpha^{2}=\frac{\int d \omega\left|S_{2}(\omega)\right|^{2}}{\int d \omega|S(\omega)|^{2}}=\tau_{e}^{2} \frac{\int d \omega \omega^{2}|S(\omega)|^{2}}{\int d \omega|S(\omega)|^{2}}=\frac{2}{3} .
$$

Close to the source and neglecting the emittance contribution, the rms betatron pulse length (coherent) is

$$
\tau_{\mathrm{rms}}=\sqrt{\frac{2}{3}} \tau_{e}
$$


[1] E. Esarey, B. A. Shadwick, P. Catravas, and W. P. Leemans, Synchrotron radiation from electron beams in plasma-focusing channels, Phys. Rev. E 65, 056505 (2002).

[2] K. Ta Phuoc, S. Corde, R. Shah, F. Albert, R. Fitour, J.-P. Rousseau, F. Burgy, B. Mercier, and A. Rousse, Imaging Electron Trajectories in a Laser-Wakefield Cavity Using Betatron X-Ray Radiation, Phys. Rev. Lett. 97, 225002 (2006).

[3] A. D. Debus, M. Bussmann, U. Schramm, R. Sauerbrey, C. D. Murphy, Z. Major, R. Hörlein, L. Veisz, K. Schmid, J. Schreiber et al., Electron Bunch Length Measurements from LaserAccelerated Electrons Using Single-Shot Thz Time-Domain Interferometry, Phys. Rev. Lett. 104, 084802 (2010).

[4] O. Lundh, J. Lim, C. Rechatin, L. Ammoura, A. Ben-Ismaïl, X. Davoine, G. Gallot, J.-P. Goddet, E. Lefebvre, V. Malka et al., Few femtosecond, few kiloampere electron bunch produced by a laser-plasma accelerator, Nat. Phys. 7, 219 (2011).

[5] O. Lundh, C. Rechatin, J. Lim, V. Malka, and J. Faure, Experimental Measurements of Electron-Bunch Trains in a Laser-Plasma Accelerator, Phys. Rev. Lett. 110, 065005 (2013).

[6] J. Van Tilborg, C. B. Schroeder, C. V. Filip, C. Tóth, C. G. R. Geddes, G. Fubiani, R. Huber, R. A. Kaindl, E. Esarey, and W. P. Leemans, Temporal Characterization of Femtosecond Laser-Plasma-Accelerated Electron Bunches Using Terahertz Radiation, Phys. Rev. Lett. 96, 014801 (2006).

[7] Y. Glinec, J. Faure, A. Norlin, A. Pukhov, and V. Malka, Observation of Fine Structures in Laser-Driven Electron Beams Using Coherent Transition Radiation, Phys. Rev. Lett. 98, 194801 (2007).

[8] A. H. Lumpkin, M. LaBerge, D. W. Rule, R. Zgadzaj, A. Hannasch, O. Zarini, B. Bowers, A. Irman, J. P. Couperus Cabadağ, A. Debus et al., Coherent Optical Signatures of Electron Microbunching in Laser-Driven Plasma Accelerators, Phys. Rev. Lett. 125, 014801 (2020).

[9] J. Wenz, S. Schleede, K. Khrennikov, M. Bech, P. Thibault, M. Heigoldt, F. Pfeiffer, and S. Karsch, Quantitative x-ray phasecontrast microtomography from a compact laser-driven betatron source, Nat. Commun. 6, 1 (2015).

[10] B. Paroli, E. Chiadroni, M. Ferrario, and M. Potenza, Analogical optical modeling of the asymmetric lateral coherence of betatron radiation, Opt. Express 23, 29912 (2015).

[11] S. Kneip, C. McGuffey, J. L. Martins, S. Martins, C. Bellei, V. Chvykov, F. Dollar, R. Fonseca, C. Huntington, G. Kalintchenko et al., Bright spatially coherent synchrotron $\mathrm{x}$ rays from a table-top source, Nat. Phys. 6, 980 (2010).

[12] B. Guo, X. Zhang, J. Zhang, J. Hua, C.-H. Pai, C. Zhang, H.-H. Chu, W. Mori, C. Joshi, J. Wang, and W. Lu, High-resolution phase-contrast imaging of biological specimens using a stable betatron x-ray source in the multiple-exposure mode, Sci. Rep. 9, 7796 (2019).

[13] S. Kneip, C. McGuffey, F. Dollar, M. Bloom, V. Chvykov, G. Kalintchenko, K. Krushelnick, A. Maksimchuk, S. Mangles, T. Matsuoka et al., X-ray phase contrast imaging of biological specimens with femtosecond pulses of betatron radiation from a compact laser plasma wakefield accelerator, Appl. Phys. Lett. 99, 093701 (2011).

[14] J. Wood, D. Chapman, K. Poder, N. Lopes, M. Rutherford, T. White, F. Albert, K. Behm, N. Booth, J. Bryant et al., Ultrafast imaging of laser driven shock waves using betatron x-rays from a laser wakefield accelerator, Sci. Rep. 8, 1 (2018).

[15] M. Mo, Z. Chen, S. Fourmaux, A. Saraf, K. Otani, J. Kieffer, Y. Tsui, A. Ng, and R. Fedosejevs, Laser wakefield generated $\mathrm{X}$-ray probe for femtosecond time-resolved measurements of ionization states of warm dense aluminum, Rev. Sci. Instrum. 84, 123106 (2013).

[16] K. Ta Phuoc, R. Fitour, A. Tafzi, T. Garl, N. Artemiev, R. Shah, F. Albert, D. Boschetto, A. Rousse, D. Kim et al., Demonstration of the ultrafast nature of laser produced betatron radiation, Phys. Plasmas 14, 080701 (2007).

[17] W. Lu, M. Tzoufras, C. Joshi, F. Tsung, W. Mori, J. Vieira, R. Fonseca, and L. Silva, Generating multi-gev electron bunches using single stage laser wakefield acceleration in a $3 \mathrm{~d}$ nonlinear regime, Phys. Rev. ST Accel. Beams 10, 061301 (2007).

[18] A. R. Rossi, A. Bacci, M. Belleveglia, E. Chiadroni, A. Cianchi, G. Di Pirro, M. Ferrario, A. Gallo, G. Gatti, C. Maroli et al., The external-injection experiment at the sparc_lab facility, Nucl. Instrum. Methods Phys. Res., Sect. A 740, 60 (2014).

[19] B. Marchetti, R. Assmann, C. Behrens, R. Brinkmann, U. Dorda, K. Floettmann, I. Hartl, M. Huening, Y. Nie, H. Schlarb et al., Electron-beam manipulation techniques in the sinbad linac for external injection in plasma wake-field acceleration, Nucl. Instrum. Methods Phys. Res., Sect. A 829, 278 (2016).

[20] N. Andreev, S. Kuznetsov, B. Cros, V. Fortov, G. Maynard, and P. Mora, Laser wakefield acceleration of supershort electron bunches in guiding structures, Plasma Phys. Controll. Fusion 53, 014001 (2010).

[21] A. Thomas, Algorithm for calculating spectral intensity due to charged particles in arbitrary motion, Phys. Rev. ST Accel. Beams 13, 020702 (2010).

[22] M. Chen, E. Esarey, C. Geddes, C. Schroeder, G. Plateau, S. Bulanov, S. Rykovanov, and W. Leemans, Modeling classical and quantum radiation from laser-plasma accelerators, Phys. Rev. ST Accel. Beams 16, 030701 (2013).

[23] V. Horný, J. Nejdl, M. Kozlová, M. Krůs, K. Boháček, V. Petržílka, and O. Klimo, Temporal profile of betatron radiation from laser-driven electron accelerators, Phys. Plasmas 24, 063107 (2017).

[24] A. Curcio, M. Bergamaschi, R. Corsini, D. Gamba, W. Farabolini, R. Kieffer, T. Lefevre, S. Mazzoni, V. Dolci, M. Petrarca et al., Diffractive shadowing of coherent polarization radiation, Phys. Lett. A 391, 127135 (2021).

[25] L. Cohen, Time-frequency Analysis (Prentice Hall PTR, Englewood Cliffs, NJ, 1995), Vol. 778.

[26] L. Guilbeau, The history of the solution of the cubic equation, Math. News Lett. 5, 8 (1930).

[27] I. Kostyukov, S. Kiselev, and A. Pukhov, X-ray generation in an ion channel, Phys. Plasmas 10, 4818 (2003).

[28] A. Curcio, M. Anania, F. Bisesto, E. Chiadroni, A. Cianchi, M. Ferrario, F. Filippi, D. Giulietti, A. Marocchino, M. Petrarca et al., Trace-space reconstruction of low-emittance electron beams through betatron radiation in laser-plasma accelerators, Phys. Rev. Accel. Beams 20, 012801 (2017).

[29] V. Horný, M. Krůs, W. Yan, and T. Fülöp, Attosecond betatron radiation pulse train, Sci. Rep. 10, 15074 (2020).

[30] A. Hofmann, The Physics of Synchrotron Radiation (Cambridge University Press, Cambridge, 2004), Vol. 20. 
[31] J. D. Jackson, Classical Electrodynamics (Wiley, New York, 1999).

[32] G. M. Garibian, On the theory of transition radiation and ionization energy losses of particle, Zh. Eksp. Teor. Fiz. 37, 527 (1959).

[33] C. B. Schroeder, E. Esarey, J. van Tilborg, and W. P. Leemans, Theory of coherent transition radiation generated at a plasma-vacuum interface, Phys. Rev. E 69, 016501 (2004).

[34] A. Curcio, M. Bergamaschi, R. Corsini, W. Farabolini, D. Gamba, L. Garolfi, R. Kieffer, T. Lefevre, S. Mazzoni, K. Fedorov et al., Noninvasive bunch length measurements exploiting Cherenkov diffraction radiation, Phys. Rev. Accel. Beams 23, 022802 (2020). 